\title{
Genomic education for the next generation of health-care providers
}

\author{
MaryAnn Campion, MS, EdD (1) ${ }^{1}$, Constance Goldgar, MS, PA-C ${ }^{2}$, Robert J. Hopkin, MD ${ }^{3}$, \\ Cynthia A. Prows, MSN, APRN ${ }^{4}$ and Shoumita Dasgupta, PhD ${ }^{5}$
}

\begin{abstract}
Historically, medical geneticists and genetic counselors have provided the majority of genetic services. Advances in technology, reduction in testing costs, and increased public awareness have led to a growing demand for genetic services in both clinical and directto-consumer spaces. Recent and anticipated changes in the workforce of genetic counselors and medical geneticists require a reexamination of the way we educate health-care providers and the means by which we provide access to genetic services. The time is ripe for rapid growth of genetic and genomic services, but to capitalize on these opportunities, we need to consider a variety of educational mechanisms to reach providers both within and
\end{abstract}

beyond the traditional genetic counseling and medical genetics sectors, including nurses, physician assistants, and nongenetics physicians. This article summarizes the educational efforts underway in each of these professions.

Genetics in Medicine (2019) 21:2422-2430; https://doi.org/10.1038/s41436019-0548-4

Keywords: genomics education; genetic services; genetics workforce

\section{INTRODUCTION}

In 2011, the Secretary's Advisory Committee on Genetics, Health, and Society issued a statement indicating that the United States had inadequate genetics education of healthcare professionals, that it was inadequate in both amount and type, and that modifications were needed in training across multiple medical specialties to ensure that health-care professionals entering the workforce are well-trained in genetics. ${ }^{1}$ Since this statement, advances in technology, reduction in testing costs, and increased public awareness have led to a growing demand for genetic services. On average, approximately ten new genetic testing products enter the market every day. ${ }^{2}$ In April 2017, the FDA began granting approval of the first direct-to-consumer tests that provide genetic risk information for certain conditions, ${ }^{3}$ and in October 2018, 23andMe received authorization to offer reports to customers on pharmacogenetics. ${ }^{4}$

Historically, medical geneticists and genetic counselors have provided the majority of genetic services. Yet, the current and projected workforce of genetic counselors and medical geneticists are not adequate to meet the growing demand for genetic services. This paper describes the current landscape of genomic education for genetic counselors, medical geneticists, nongenetics physicians, nurses, and physician assistants; differentiates the variables impacting the future of genetic service delivery; and encourages readers to apply the knowledge gained to personally contribute to the genomic education of health-care providers and thus to growth and access of genetic services. While the scope of this article is limited to the five groups listed above, the authors acknowledge the important role of other clinical and scientific partners in this endeavor.

\section{GENETIC COUNSELORS}

As of this writing, there are over 4000 certified genetic counselors in the United States. ${ }^{5}$ Although the genetic counselor workforce has grown by $88 \%$ in the past 10 years, a 2016 supply and demand report confirmed that the United States was experiencing a shortage of genetic counselors engaged in direct patient care. ${ }^{6}$ Based on the identified gap, the Genetic Counselor Workforce Working Group (GCWFWG) was established in 2013 with representation from the National Society of Genetic Counselors (NSGC), the Accreditation Council for Genetic Counseling (ACGC), the Association of Genetic Counseling Program Directors (AGCPD), and the American Board of Genetic Counseling

${ }^{1}$ Department of Genetics, Stanford University School of Medicine, Stanford, CA, USA; ${ }^{2}$ Department of Family and Preventive Medicine, Division of Physician Assistant Studies, University of Utah School of Medicine, Salt Lake City, UT, USA; ${ }^{3}$ Cincinnati Children's Hospital Medical Center, Division of Human Genetics and University of Cincinnati College of Medicine, Department of Pediatrics, Cincinnati, OH, USA; ${ }^{4}$ Divisions of Human Genetics and Patient Services, Children's Hospital Medical Center, Cincinnati, OH, USA; ${ }^{5}$ Department of Medicine, Biomedical Genetics Section, Boston University School of Medicine, Boston, MA, USA. Correspondence: Shoumita Dasgupta (dasgupta@bu.edu) 
(ABGC), with the American Society of Human Genetics (ASHG) joining in 2016. The GCWFWG identified existing barriers to expansion of the genetic counselor workforce and created the 2017-2019 Workforce Strategic Plan with actionable strategies to address these barriers. The Strategic Plan includes three primary areas of focus (genetic counselor training, clinical supervisors, and public awareness/value), as well as a roadmap that aligns each intended outcome with the skills and resources of the member organizations. ${ }^{7}$

AGCPD is addressing the workforce by increasing the number of training programs and expanding existing programs, all while ensuring the maintenance of quality and rigor. The number of accredited programs in North America has grown by $31 \%$ in the past two years compared with a much slower expansion of approximately $10 \%$ over the preceding decade. $^{8}$ In addition, there are at least another 16 programs in development, ${ }^{8}$ indicating a growth potential of $35 \%$. Meanwhile, the number of training slots has doubled over the past 10 years, with an $18 \%$ increase in 2017 alone (unpublished data, AGCPD, 2018).

In addition to the efforts of AGCPD, ACGC is continuously evaluating best practices in accreditation and evaluation, as well as the structures available to support a growing number of programs. For example, the Clinical Training Assessment Taskforce was formed to explore how other health professions evaluate trainees' achievement of clinical competencies and to consider competency-based assessment guidelines for the clinical training of genetic counseling students. ACGC also has a Standards Committee, which conducted a survey of genetic counselor education stakeholders in January 2018 to adapt training standards to reflect current practice. This data was analyzed and incorporated, with final revisions of the Standards of Accreditation anticipated during summer 2019. ${ }^{9}$ Notably, a proposed change to the Standards is to eliminate the position of the Medical Director in GC program leadership. This change would not be at the expense of having experienced providers and educators in leadership roles for genetic counseling programs, as those skills would still be required of Master's in Genetic Counseling-trained program directors, with a minimum of six years of professional genetic counselor experience, course administration, and supervision and assessment experience. Rather, this reflects the difficulty in securing protected time from medical geneticists for these roles when there is an acute shortage of these providers in general. Until the workforce can be increased, genetic counseling programs should identify an affiliated clinical geneticist who at minimum can serve on their curriculum committee. In addition to the didactic needs that these individuals would address, professional education requires clinical rotations supervised by genetics experts. Clinical genetics professional societies might identify clinical genetics sites that do not yet take trainees on a regular basis or identify expert clinical geneticists willing to serve remotely to respond to difficult diagnostic or management questions. Some form of academic recognition or consultant reimbursement would be required for these remote services and away rotations.
Meanwhile, ABGC has their own efforts underway focused on credentialing. In 2015, ABGC formed the Examination Eligibility Taskforce (EETF), which was charged with evaluating the exam eligibility requirements and looking into expanding eligibility through additional pathways. The EETF's 2016 report contained seven recommendations, one of which was to develop a pathway for internationally trained genetic counselors who are recognized or certified by an agency approved by ABGC (e.g., European Board of Medical Genetics, Human Genetics Society of Australasia) to sit for the examination. The rollout of this new pathway began in January $2018 .^{5}$

While these groups are expanding training programs and counselor pipelines, the individual training programs are updating their curricula to ensure that students are being prepared for the future. Results from the 2018 NSGC Professional Status Survey indicate that $25 \%$ of respondents work in nondirect patient care positions, such as research, industry, laboratory support, business development, marketing, consulting, clinical coordination, supervision, public health, public policy, and advocacy. In addition, 32\% of respondents reported working remotely, either some or all of the time. ${ }^{10}$ Therefore, programs are including more training in emerging markets, as well as alternative service delivery models, such as telephone and video-based counseling. To maximize flexibility and resources, programs are also incorporating more innovative training strategies, such as utilizing standardized patients, creating remote training opportunities, developing interprofessional educational activities, providing blended and distance learning, and integrating creative models of supervision. $^{11}$

In addition to supporting the efforts discussed above, NSGC also advocates for state licensure for genetic counselors, with 26 states having passed licensure bills, and for federal legislation that would allow the Centers for Medicare and Medicaid Services (CMS) to recognize genetic counselors as providers, thus allowing genetic counselors to bill CMS for genetic counseling services. Genetic counselors are often considered to provide a cost-saving service in that they help to identify patient concerns and issues unique to genetic and inherited conditions. Additionally, genetic counselors are able to identify the correct test based on personal and family history, thereby helping to minimize unnecessary testing. Therefore, recognition as a CMS provider is anticipated to result in quality anticipatory care for patients with a genetic contribution to disease. The value-added service is expected to result in increased reimbursement for genetic counseling services across insurance providers. NSGC is also expanding collaborations with other clinical partners. For example, several nursing and physician organizations have reached out to NSGC requesting participation in their creation of genetics certification and educational programs (personal communication, NSGC, 2018). While participation does not imply endorsement, these invitations address the issue of access and the idea that all genetic service providers share a common goal. 


\section{MEDICAL GENETICISTS}

For physician residency programs for genetic services, the workforce challenges are daunting. ${ }^{12}$ There is increasing recognition that understanding the genetic basis of disease is essential to best care, and yet historically many patients with genetic conditions have not been evaluated by a provider with expertise in genetics due to barriers such as geographic distance, long wait times, inadequate insurance coverage, and ineffective triage and referral systems. ${ }^{13}$ At this time, there are approximately 1583 board-certified geneticists in the United States, ${ }^{14}$ which represents approximately $0.1 \%$ of practicing physicians. On average a geneticist sees 1350 patient visits per year. ${ }^{15}$ This would provide up to 1.35 million encounters per year, while the estimated need is up to 3 million encounters per year based on the prevalence of rare disorders. ${ }^{16}$ This gap in accessible genetics services has increased wait times, with more than $30 \%$ of geneticists responding to a 2015 survey reporting new patient, nonemergency wait times of more than three months, compared with only $10 \%$ of respondents to a similar survey in 2005. In addition, $62 \%$ of geneticists reported that their practices were nearly full, with $9 \%$ indicating that they were not accepting new patients, in comparison to only $5 \%$ of geneticists reporting full practices in $2005 .{ }^{13}$ In March 2018 there were 184 postings for "clinical geneticist" positions listed on the ACMG website jobs board, ${ }^{17}$ and yet the expected number of graduates in 2019 and 2020 is anticipated to be 45 and 37, respectively. ${ }^{18}$ There is clearly a critical need for more well-qualified providers in the short term and for a strategic plan to increase the capacity to attract and train new generations of providers.

It is ironic that the supply of new practitioners is critically low at a time when the knowledge base and tools used in the field are accelerating discovery and enhancing our ability to both establish diagnoses and treat patients with genetic conditions. The training for a physician geneticist has traditionally included a residency in a primary care field (most commonly pediatrics, internal medicine, and obstetrics/ gynecology) followed by a second residency in genetics that was often referred to as a fellowship. This took at least 5 years after graduation from medical school. More recently there has been increasing interest in combined programs (pediatrics/ genetics, maternal-fetal medicine/genetics, or internal medicine/genetics), which have recently been reduced from 5-year to 4-year programs in an attempt to attract more trainees. The percentage of trainees in the combined programs has increased in the past several years to approximately $30 \%$ $\left(\right.$ ref. ${ }^{18}$ ). However, the hoped-for increase in total applicants has not materialized. In 2017-2018, a total of 83 training positions were posted including both the traditional categorical pathway and combined programs, but only 37 positions $(45 \%)$ were filled. $^{18}$ Becoming a board-certified geneticist requires a longer period of training than a pediatrician or internal medicine specialist, but the income potential is decreased with the added training. ${ }^{19}$ The discrepancies in income will need to be addressed, but that is likely to require action from the national organizations.
One key challenge in increasing interest in clinical genetics is that under current billing regulations, geneticists rarely generate enough revenue to cover the cost of their employment. Adjusting billing/compensation practices to allow providers of genetic services to generate profit or break even would allow for increased hiring when needed. This is a challenge that affects all providers of genetic services. Both the ACMG and NSGC have committees working on improving coverage for genetic services; these groups should work together and include other fields with a vested interest in genetics to maximize impact of requests for improved coverage. Successful negotiation will also require collecting data demonstrating the value of genetic services in improving health outcomes.

In terms of recruitment into the specialty, the reasons for the lack of applicants are not well documented, but several factors within medical training and current medical genetics practices have been suggested. As will be outlined below, exposure to didactic genetics content in medical school, especially during the clinical years, is limited. ${ }^{20}$ Even among trainees who choose to specialize in medical genetics, $32 \%$ have not had a medical genetics elective prior to entering the medical genetics residency. ${ }^{12}$ To change students' exposure to genetics in medical training, genetics professionals must increase their involvement in care management of multisystem genetic conditions (e.g., increasing outpatient clinic slots for follow-up management, admitting genetics patients to genetics service, participating in inpatient daily rounds for patients with genetic disorders who are not on genetics service, and formalizing a role in patient-centered medical homes for patients with genetic disorders. ${ }^{21}$ At the time of this writing, a search for clinical care on the ACMG or ASHG websites returned information only for diagnostic testing. There is essentially no description of a role in management once a diagnosis is established, nor is there an indication that monitoring, interventions, or management of genetic syndromes impact the outcome for affected patients. ${ }^{17,22}$ Transitioning from a focus on diagnosis to a focus on providing care guided by natural history or molecular pathology revealed by an accurate diagnosis will build evidence for improved outcomes related to medical genetics practice and will also increase visibility among trainees in these settings on their required rotations. The impact of this approach would be greatly increased if a national data repository that facilitates registry-based studies for genetic conditions was created. This type of large-scale outcome data is particularly important for the rarest condtions.

Because most medical students anticipate a career with direct involvement in improving outcomes, the role of genetic medicine in direct patient care needs to be emphasized during medical school and residency training for all physicians and specialties. Medical geneticists could also look for more targeted opportunities to mentor those interested in genetics to ensure that potential trainees appreciate the fascinating and dynamic qualities of this field. Lastly, rather than perpetuating the idea that genetic testing is scary and/or dangerous, the 
genetics community would benefit from focusing their message on real examples of how diagnostic testing has changed treatment and outcomes and how predisposition testing has led to successful anticipatory management using detection of previously unrecognized yet treatable disease. In combination with ACMG and ASHG's existing outreach activities designed to increase awareness of and interest in genetics, additional initiatives such as the ones described above could create the culture change that is necessary to establish a sustainable workforce of medical geneticists.

With the current shortage of clinical geneticists there is a clear challenge because the already stressed providers will need to be engaged in any of the potential solutions. Optimizing efficiency with increased use of telehealth or other models to increase access to providers, and developing strong collaborations with nongeneticists who are interested in genetics, can facilitate application of genetic information in spite of currently limited numbers of providers. In addition, there are some recent efforts to integrate links to management guidelines in electronic medical records to help access current standards. ${ }^{23}$ This simplified access to current management recommendations could greatly facilitate improved care and decrease the time required from a limited number of providers.

\section{NONGENETICS PHYSICIANS}

Given the demonstrated dearth of genetic specialists both practicing as physicians and in the residency pipeline, the need to improve recruitment into the specialty is acute. Even if the existing training pathways were to fill to capacity, the current pipeline is inadequate to meet the expected need. Furthermore, given the scarcity of clinical geneticists in practice, trainees are not gaining meaningful exposure to role models in the field during their medical training. Genetic technology is developing at such an incredible pace that advances in genome sequencing technologies dwarf comparisons with Moore's law (which describes the pace of innovations in computing power). ${ }^{24}$ In this context, it is challenging to imagine that nonspecialists outside of full-time training programs will be able to keep up with the full extent of these developments in genetics and genomics. As such, the training paradigms for medical students take on increased significance, since medical students are considering whether their career paths might include specialization in medical genetics during their MD studies.

When considering the factors that may impact tracking of medical students into medical genetics residency programs, it is important to consider how the curriculum is designed with respect to its medical genetics content. Although medical school curricula take on many unique structures, certain common elements can be used to describe curricular design. For instance, medical schools frequently have preclinical and clinical phases of education; while there is some intermingling of these phases, it tends to be more heavily preclinical in the early stages of training, and more focused on clinical practice during the later stages of training. In this context, it is troubling to note that the majority (75\%) of formal exposure to genetics content in medical school curricula comes in the first year of medical school, ${ }^{20}$ at a time that is frequently perceived by trainees as focused on basic science content with limited relevance to clinical practice. Similarly, it was found that only $26 \%$ of respondents had any medical genetics content placed in the clinical years of training. ${ }^{20}$ To address this gap, developing educational tools to demonstrate the clinical application of genetics would be very helpful, and there are abundant examples available. The major hurdle for this is protecting time for the clinicians who currently provide this type of care for educational activities. Fortunately, nongeneticists who have an interest in genetics can actively participate in addressing this need, as can genetic counselors and other providers who see patients with genetic diseases.

Another key aspect of medical curricular design is the identity of the content as presented to the trainees. This can be traced back to whether courses are offered in a more traditional discipline-based curriculum versus in an integrated curriculum. In discipline-based curricula, genetics content is easily identifiable as its own topic, albeit generally a basic science one. However, medical curricula are trending toward an integrated curriculum structure ( $75 \%$ of curricula) where genetics is woven as a thread throughout many systems-based courses. This structure, while it emphasizes clinical application, threatens a loss of identity of genetics content beyond the description of a series of rare diseases because genetics typically only represents $2 \%$ of preclinical curricular time. ${ }^{20}$

Taken together, the relative lack of genetics content during the clinical years and a dilution of its identity as a discipline have significant potential negative consequences. Importantly, these curricular trends also limit exposure to role models in medical genetics, especially during clinical stages of training. These curricular structures threaten to further reduce the visibility of specialized training opportunities in clinical genetics. It follows that this may negatively impact recruitment into this critical specialty, as mentioned above. ${ }^{25}$

Clearly, the challenges facing clinicians in trying to adopt genomic medicine approaches cannot be solved by specialists alone. The majority of MD graduates will go on to practice in specialties outside of medical genetics, but if they bring a baseline genomic literacy to their clinical practice from their recent training, they can begin to provide reverse mentoring to their care team colleagues. ${ }^{26}$ In this model where recent MD graduates are advocates for genomic medicine in their clinics, core curriculum in medical schools must prepare the nonspecialist to implement relevant genomic medicine principles into their practice. Therefore, it is critical to make the case that genomic medicine content should be valued, protected, and even expanded as curricula grow and change over time. A peer-reviewed core curriculum designed with the input of educators from the Association of Professors of Human and Medical Genetics ${ }^{27}$ can provide guidance on which topics are best emphasized in a required medical school curriculum, both in preclinical and clinical phases of training, for the general graduate. Ideally, people who write questions 
for licensing exams will also begin to consider connections with genetic principles as they prepare these assessments. Beyond inclusion in the formal curriculum, educators should also consider creative ways to increase exposure to medical genetics, including initiation of student interest groups, promotion of summer experiences like the ACMG Summer Scholars Program, ${ }^{28}$ development of tracks or electives for students who would like further exposure to this growing field, and creation of opportunities for personalized, donor, or anonymous genome analysis. ${ }^{29} \mathrm{~A}$ range of educational strategies around topics in genomic medicine have been piloted both in formal and informal medical educational settings, and may serve as inspiration for development of additional programs in this space. ${ }^{30}$

\section{NURSES}

For the purposes of this paper, we focus on genomics education for licensed registered nurses (RNs) and advanced practice registered nurses (APRNs). There are more than 2.8 million employed $\mathrm{RNs}^{31}$ and 270,000 APRNs ${ }^{32}$ in the United States. While all APRNs must have a graduate degree in nursing, ${ }^{33}$ education preparation for practicing RNs may be a two-year associate degree, four-year baccalaureate degree, or less frequently a three-year diploma in nursing. ${ }^{34} \mathrm{RNs}$ provide patient education, community education, care coordination, and psychosocial support, and they administer and monitor medications and therapies for patients across the lifespan. In contrast, APRNs assess, diagnose, and manage patient health problems; order and interpret tests and procedures; and prescribe medications consistent with their education and certification as a nurse practitioner, clinical nurse specialist, nurse midwife, or nurse anesthetist. ${ }^{35}$

$\mathrm{RN}$ and APRN education programs may or may not have genetic/genomic content in their curricula, and those programs that do vary considerably in the amount and type of genetics content that is covered. This inconsistency exists despite a long history of content recommendations in the $1960 \mathrm{~s},{ }^{36}$ a resurgence of recommendations in the $1980 \mathrm{~s},{ }^{37}$ and ongoing National Institutes of Health (NIH) and Health Resources and Services Administration (HRSA) funded efforts to provide education since the $1990 \mathrm{~s}^{38-43}$ to increase genetics content within nursing curricula. Although increases in genetics content in nursing curricula have been measured in schools where faculty have participated in intensive continuing education, ${ }^{38}$ national surveys continue to reflect limited genetics knowledge among nursing faculty. ${ }^{44}$ Therefore, even if genetics and genomics content is taught within science courses, nursing faculty may not be prepared to translate the science in their didactic and clinical courses.

Nursing was a leader in clearly delineating roles, responsibilities, and competencies related to genetics. The first Statement on the Scope and Standards of Genetics Nursing Practice was published in 1997 and most recently updated in $2016 .{ }^{45}$ The statement describes the roles and responsibilities of RNs and APRNs specializing in genetics at the basic or advanced levels. Genetics and genomics competencies for all nurses were developed through a consensus process with leaders from numerous nongenetics nursing specialty organizations, academic programs, as well as nursing leaders in federal agencies and the International Society of Nurses in Genetics (ISONG). ${ }^{46}$ The competencies were subsequently updated to include outcome indicators. ${ }^{47}$ A similar process was used to establish genetics and genomics competencies for all nurses with graduate degrees. ${ }^{48}$ The American Association of Colleges of Nursing (AACN) recognized the importance of the competencies and adopted select competencies in the Essentials for Baccalaureate Education in Nursing and the Essentials of Graduate Education in Nursing. ${ }^{49}$ The Essentials documents are used by the Commission on Collegiate Nursing Education (CCNE) when developing criteria for accreditation of baccalaureate and graduate level nursing programs.

While progress has been made to establish a foundation from which to guide nursing education programs, significant regulatory barriers persist. During the accreditation process, CCNE assesses education programs that offer master's and baccalaureate level nursing degrees for the presence of genetics content or a plan for implementing genetics content before the next cycle. The Accreditation Commission for Education in Nursing accredits all types of nursing education programs but evidence of assessing curricula for genetics content could not be found in the literature. The $\mathrm{RN}$ licensure exam is informed by regularly updated knowledge assessments. In the 2014 National Council for State Boards of Nursing's knowledge assessment, ${ }^{50}$ newly licensed RNs, clinical nursing supervisors, and nurse educators were asked to rank 253 knowledge statements according to the question, "How important is the possession of this knowledge by a newly licensed RN for safe and effective professional practice, regardless of specific practice setting?" Using the document search function, "genetics," "genomics," "gene," and "inheritance" were not found in the 253 items nor were they mentioned in the 140-page report. Based on the knowledge assessment items, it can be predicted that genetics/genomics content will continue to be absent from $\mathrm{RN}$ licensure examinations.

Because nursing education curricula may or may not contain genomics content, considerable effort has also been focused on creating and delivering genomics continuing education for practicing nurses. Two authors of this paper (R. J.H. and C.P.) were involved in the creation of a multifaceted Genetics Education Program for Nurses (GEPN) that was initiated with NIH and HRSA funds and sustained for 20 years through registration fees. At the time of this writing, over 17,000 nurses, nursing faculty, and nursing students had completed one or more of the continuing education offerings. The National Council of State Boards of Nursing provided funds to evaluate a genomics education intervention that focused on nurse educator and hospital administrator dyads at 21 Magnet hospitals. ${ }^{51}$ Finally, the ISONG has been providing a variety of genetics continuing education since its incorporation in $1989 .^{46}$ 
Even if only $1 \%$ of the nursing workforce could be motivated to achieve adequate genetics continuing education, that would amount to nearly 30,000 professionals that could significantly contribute to improving access to genomic services. As noted earlier in this paper, clinical genetics is moving beyond diagnosis and counseling to include care management as well as prescribing and treating patients with a growing number of condition-specific therapeutic options. This expansion of clinical genetics opens the doors to RNs who can coordinate clinics, administer medications, and provide patient/family/community education about therapies and how to monitor for and respond to adverse drug reactions. While APRN graduate training is not specific to most medically recognized specialties and subspecialties, APRN employment has notably grown in a wide variety of specialty practices. ${ }^{52}$ To promote APRN success in diverse specialty areas, residencies, fellowships, and mentorship programs have been developed to prepare APRNs for full scope of practice. ${ }^{53}$ Likewise, APRNs do not need to be experts in genetics when hired into the specialty. While development of a nationally recognized, accessible curriculum and fellowship plan for new APRNs in genetics would be ideal, such resources do not currently exist, leaving clinical genetics practices to provide role-specific on-the-job education and training. On-the-job training might focus on natural history, management, and treatment strategies for a subset of patients who may share a specific genetic diagnosis such as neurofibromatosis, Ehlers-Danlos syndrome, or a group of diagnoses such as heritable cancers or cardiovascular disorders. By managing a caseload of genetics patients who require ongoing follow-up and medication management, APRNs can free up clinical geneticists to focus on new visits and managing the care of patients with complex rare disorders, thus reducing the unreasonable wait time for clinical genetics appointments.

\section{PHYSICIAN ASSISTANTS}

Physician assistants (PAs) are a rapidly growing profession with over 130,000 nationally certified PAs in the United States. ${ }^{54}$ They are medical professionals who diagnose illness, develop and manage treatment plans, prescribe medications, and often serve as a patient's principal health-care provider. With thousands of hours of medical training, PAs are versatile, collaborative, and practice in every medical setting. ${ }^{54}$ PAs are taught in the medical model with a generalist education base from their inception. They have the capability to move from specialty to specialty throughout their careers, and are able to respond nimbly to workforce needs as they evolve, improving health-care access and quality. PAs practice in all specialty areas where they expand and deepen skills in the specific professional activities of their work settings. The PA practice model is collaborative by definition. All PAs currently have shared practice arrangements with supervising physicians; the supervisory relationship with the physician and scope of practice is regulated at the level of the individual state medical licensing authority.
The PA community published a survey and needs assessment regarding genetic education in PA programs in 2007, shortly after the Accreditation Review Commission on Education for the Physician Assistant (ARC-PA) created a new accreditation standard that mandated teaching "the genetic and molecular mechanisms of health and disease" in all PA programs. ${ }^{55}$ In 2007, the publication "Establishing Essential Physician Assistant Clinical Competencies Guidelines for Genetics and Genomics" provided core competencies to guide PA educators in teaching genetics. ${ }^{56}$ In 2016, an updated set of PA Genomic Competencies was published using the Accreditation Council for Graduate Medical Education (ACGME) domains of competencies already adopted by PA organizations. ${ }^{57}$ The revised genomic competencies were designed to more generally anticipate future clinical genetic activities and were restructured to enhance collaborative medical education for PA students and providers-with an emphasis on interprofessional, teambased medicine. PA genetic education occurs almost wholly in the classroom during the didactic year and is variable across programs. During the clinical education portion of PA training, genetic concepts are infrequently taught or reinforced by physician or PA preceptors, so their importance and uptake continue to be limited.

Although there has not been a survey of practicing PAs, their specialties, and use of genomics in practice, the American Academy of Physician Assistants sponsors a Special Interest Group in Genetics for practicing PAs; ${ }^{58}$ in parallel, a group of PA educators with special interest in genetics exists nationally and has plans for such a survey. In addition, NIHsponsored programs exist to help clinically practicing PAs and advanced nurse specialists complete additional genetics training in oncology. ${ }^{59}$

Many of the specialties in which PAs work include those that now require more concentrated learning in genetics and genomics, including pediatrics, obstetrics, oncology, and increasingly cardiology. In addition to specialty-specific training, PAs currently are hired to partner with MD geneticists who provide on-the-job training to help reduce the long waiting times for specific groups of patients needing to be seen. This would include "practice agreements" between the supervising physician partner and the PA, as state laws dictate. Although PA postgraduate programs exist in any number of specialties across the country, currently there are no certificate or postgraduate programs in medical genetics. However, postgraduate or dual-degree programs could be conceived in this area based on appropriate institutional infrastructure, expertise, and support in medical genetics for interested PAs.

With appropriate skill acquisition occurring in the work setting, PAs educated in the medical model are currently capable of impacting workforce needs in a multitude of areas, including medical genetics. Because of fewer barriers to PAs changing specialties during their career, their continual acquisition of skills of the setting in which they work makes the profession an attractive model for furthering genetics and 
genomics in clinical practice. Learning with their physician partners how to practically apply clinical genomics strategies to patient care in one setting could then be adapted and applied to subsequent specialties.

Though PA genetics education presently has unfortunately not been emphasized or reinforced clinically, as noted with other medical professions, it is clear that the need for genetics services for patients will only increase. Depending on the setting, this can be accomplished with on-the-job or specific postgraduate medical genetics training, but always collaboratively with partner physicians. PA and other health professional team members' facility and expertise in clinical genomics needs to rise, so the level of care they provide to patients will be personalized to provide the preventive, diagnostic, and therapeutic management that is crucial to their patients' optimal health. ${ }^{57}$

\section{CONCLUSIONS}

With the reality of clinical genomic technology developments far outpacing the training of specialist and nonspecialist providers, we offer the following suggestions for readers to get involved and to begin to address this gap:

- Early education: In 2013, several states adopted the Next Generation Science Standards for K-12 Public Schools. ${ }^{60}$ Therefore, presentations to these groups can be designed around the five genetics-related standards for middle school (grades 6-8) or the ten genetics-related standards for high school (grades 9-12). The Jackson Laboratory also offers a week-long professional development course on Teaching the Genome Generation ${ }^{61}$ for high school teachers; tuition is free, and a stipend is provided for teachers from New England.

- Undergraduate education: Readers whose educational interests align with college curricula should consider attending ASHG's Undergraduate Faculty Genetics Education annual workshop. These workshops are designed to explore innovative and active pedagogies that are effective in teaching genetics concepts related to current, socially relevant themes. Similarly, readers working in a research capacity should consider connecting with prospective students through the Genomics Undergraduate Partnership. $^{62}$

- Graduate education: The ASHG website ${ }^{63}$ provides a list of master's and doctoral programs that can be targeted to find students whose interests might align with a career in genetics.

- Professional education: For those interested in education for established health-care providers, the Genetics and Genomics Competency Center ${ }^{64}$ serves as an online repository of educational materials for educators, students, and clinicians. These materials, which are mapped to discipline-specific genomic competencies, allow educators to tailor their message to meet the needs of a specific audience based on their field of practice. In addition, the Association of Professors of Human and Medical
Genetics ${ }^{65}$ holds an annual meeting where stakeholders in clinical genetics education (medical, residency, lab program, and interprofessional educators) meet to discuss trends, develop shared educational resources to contribute to their Genetics Education Resource Exchange, and carry out national educational initiatives in this space.

- Continuing education: There are many resources available for readers who want more training in scientific pedagogy. The Center for the Integration of Research, Teaching, and Learning $^{66}$ is a consortium of 41 research universities in the United States and Canada that has a massive open online course entitled Advancing Learning through Evidence-Based STEM Teaching, which has significant coverage of genetics. The concepts and strategies covered in this course can be extended to any group of adult learners. Additional resources are available through the Jackson Laboratory ${ }^{67}$ and the American Medical Association, ${ }^{68}$ which provide a variety of complimentary, interactive webinars and online modules, many of which are available for continuing education credit. Additional efforts should also be made to provide similar opportunities for engaging in genetics training by members of the allied health professionals, perhaps through continuing education, postgraduate, or certificate programs.

In closing, we encourage educators and leaders from each of the professions discussed above to continue conversations about their complementary scopes of practice. Given the shortage of providers, the authors recognize the significant clinical demands on their time. Toward this end, we advocate for increased collaboration across disciplines. For example, in 2013, the National Human Genome Research Institute, together with 23 professional societies, 15 other institutes at the National Institutes of Health, and other organizations interested in physician education, developed the Inter-Society Coordinating Committee for Physician Education in Genomics (ISCC) to develop and share best practices in the use of genomics in medicine. The ISCC developed a framework for development of genomics practice competencies that may serve as a starting point for formulation of competencies for physicians in various medical disciplines. This initiative addressed the concept of entrustable professional activities (EPAs), which are defined by medical educators as those professional activities that together constitute the mass of critical elements that operationally define a profession. ${ }^{69}$ The Competencies Working Group identified five EPAs that comprise a basic set of genomic skills (family history, genomic testing, treatment based on genomic results, somatic genomics, and microbial genomic information), in hopes that these EPAs would provide a starting point for development of more specific guidelines for individual medical disciplines, recognizing that some EPAs may be unnecessary or require modification for various disciplines. ${ }^{70}$ Although the ISCC effort was initially aimed at physicians, the group has expanded ${ }^{71}$ to include additional 
clinical partners and serves as an example for taking a more inclusive and powerful approach to addressing future access to genetic services.

In addition to increased interprofessional and interspecialty efforts, to leverage the expertise of the available clinical geneticists, service and educational activities in this space need to be valued by genetics chairs and chiefs. This would include utilizing educational value units to offset relative value unit (RVU) targets, release time to do outreach work with trainees or to develop specialty electives, inclusion of these activities toward promotion and tenure, and use of senior trainees themselves as near peer mentors to improve tracking into medical genetics. Importantly, these elements will be most effective if we can engage the broader genetics community to give trainees the exposure they will need to truly embrace the exciting direction genomic medicine promises to take us all.

\section{ACKNOWLEDGEMENTS}

The authors thank Shannan Dixon for her expert help and suggestions with the manuscript.

\section{DISCLOSURE}

The authors declare no conflicts of interest.

Publisher's note: Springer Nature remains neutral with regard to jurisdictional claims in published maps and institutional affiliations.

\section{REFERENCES}

1. Secretary's Advisory Committee on Genetics, Health, and Society. Genetics education and training report. 2011. http://oba.od.nih.gov/ oba/SACGHS/reports/SACGHS_education_report_2011.pdf. Accessed 23 November 2018.

2. Phillips K, Deverka P, Hooker G, Douglas M. Genetic test availability and spending: where are we now? Where are we going? Health Affairs. 2018:37:710-716.

3. U.S. Food \& Drug Administration. FDA allows marketing of first direct-toconsumer tests that provide genetic risk information for certain conditions. 2017. https://www.fda.gov/newsevents/newsroom/ pressannouncements/ucm551185.htm. Accessed 23 November 2018.

4. 23andMe. 23andMe Granted The First and Only FDA Authorization For Direct-to-Consumer Pharmacogenetics Reports. https://blog.23andme. com/tag/pharmacogenetics/. Accessed 23 November 2018.

5. American Board of Genetic Counseling. 2018. http://www.abgc.net. Accessed 11 February 2019.

6. Hoskovec J, Bennett R, Carey M, et al. Projecting the supply and demand for certified genetic counselors: a workforce study. J Genet Couns. 2017:27:16-20.

7. National Society of Genetic Counselors. Genetic Counselor Workforce Working Group. Strategic Plan, 2017-2019. 2018. https://www.nsgc. org/d/do/7603. Accessed 23 November 2018.

8. Association of Genetic Counseling Program Directors, Program Directory. 2018. https://agcpd.org/ProgramDirectory.aspx. Accessed 23 November 2018.

9. Accreditation Counsel for Genetic Counseling, Standards of Accreditation. 2019. http://www.gceducation.org/standards-ofaccreditation/. Accessed 5 April 2019.

10. National Society of Genetic Counselors. Professional Status Survey, 2018. https://www.nsgc.org/d/do/8436. Accessed 23 November 2018.

11. Riconda D, Grubs R, Campion M, Cragun D. Genetic counselor training for the next generation: where do we go from here? Am J Med Genet C Semin Med Genet. 2018;178:38-45.
12. Cichon $M$, Feldman GL. Opportunities to improve recruitment into medical genetics residency programs: survey results of program directors and medical genetics residents. Genet Med. 2014;16:413.

13. Maiese DR, Keehn A, Lyon $M$, et al. Current conditions in medical genetics practice. Genet Med. 2019 Jan 28. https://doi.org/10.1038/ s41436-018-0417-6 [Epub ahead of print].

14. American Board of Medical Genetics and Genomics. Number of ABMGG Certified Specialists in Medical Genetics and Genomics, 2017. http://www. abmgg.org/pdf/Statistics\%20for\%20Webpage.pdf. Accessed 23 November 2018.

15. Sukenik-Halevy R, Ludman MD, Ben-Shachar S, Raas-Rothschild A. The time-consuming demands of the practice of medical genetics in the era of advanced genomic testing. Genet Med. 2016;18:372-377.

16. Genetic and Rare Diseases Information Center. FAQs about rare diseases. 2017. https://rarediseases.info.nih.gov/diseases/pages/31/faqs-about-rarediseases. Accessed 19 January 2019.

17. American College of Medical Genetics and Genomics. 2018. http://www. acmg.net. Accessed 23 November 2018.

18. National Resident Matching Program. Results and data: specialties matching service 2018 appointment year. 2018 main residency match. Washington, D.C.: 2018.

19. Salary.com. 2018. https://www1.salary.com. Accessed 23 November 2018.

20. Plunkett-Rondeau J, Hyland K, Dasgupta S. Training future physicians in the era of genomic medicine: trends in undergraduate medical genetics education. Genet Med. 2015;17:927-934.

21. Schaefer GB, Larson IA, Bolick J, Williamson-Dean L. What is the role of clinical genetics in the patient-centered medical home?: a commentary from the Medical Home Workgroup of the Heartland Regional Genetics and Newborn Screening Collaborative. Genet Med. 2016;18:440-442

22. American Society of Human Genetics. 2018. http://www.ashg.org. Accessed 23 November 2018.

23. Santoro SL, Bartman T, Cua CL, et al. Use of electronic health record integration for Down syndrome guidelines. Pediatrics. 2018;142: e20174119.

24. Hayden E. Technology: the $\$ 1,000$ genome. Nature. 2014;507:294-295.

25. Hyland K, Dasgupta S. Medical genetics and genomics education and its impact on genomic literacy of the clinical workforce. Genet Med. 2019;21:1259-1260.

26. Hyland K, Garber K, Dasgupta S. From helices to health: undergraduate medical education in genetics and genomics. Pers Med. 2018 Nov 29; https://doi.org/10.2217/pme-2018-0081 [Epub ahead of print].

27. Hyland K, Dasgupta S, Garber K, et al. Medical school core curriculum in genetics. Association of Professors of Human and Medical Genetics. 2013. http://www.aphmg.org/pdf/med-competencies.pdf. Accessed 23 November 2018.

28. ACMG Foundation for Genetic and Genomic Medicine. Summer Genetics Scholars Program. http://www.acmgfoundation.org/ACMGF/ What-We-Do/Summer-Scholars/ACMGFMhat/Summer_Scholars.aspx. Accessed 19 January 2019.

29. Garber KB, Hyland KM, Dasgupta S. Participatory genomic testing as an educational experience. Trends Genet. 2016;32:317-320.

30. Rubanovich C, Cheung C, Mandel J, Bloss C. Physician preparedness for big genomic data: a review of genomic medicine education initiatives in the United States. Hum Mol Genet. 2018;27(R2):R250-R258.

31. National Council of State Boards of Nursing. The nursing regulatory environment in 2018: issues and challenges. J Nurs Regul. 2018;9:52-65.

32. American Association of Nurse Practitioners. NP fact sheet. https://www aanp.org/about/all-about-nps/np-fact-sheet. Accessed 8 February 2019.

33. American Association of Colleges of Nursing. http://www.aacnnursing. org. Accessed 19 January 2019.

34. National League for Nursing. Nursing Programs. 2018. http://www.nln. org/newsroom/nursing-education-statistics/nursing-programs. Accessed 23 November 2018.

35. National Council of State Boards of Nursing. APRNS in the U.S. https:// www.ncsbn.org/aprn.htm. Accessed 19 January 2019.

36. Brantl V, Esslinger P. Genetics-implications for the nursing curriculum. Nurs Forum. 1962;1:90-100.

37. Anderson EH. Nursing education in genetics. Washington, D.C.: National Maternal and Child Health Clearinghouse; 1985.

38. Prows CA, Hetteberg C, Johnson N, et al. Outcomes of a genetics education program for nursing faculty. Nurs Educ Perspect. 2003;24:81-85. 
39. Prows CA, Hetteberg C, Hopkin RJ, et al. Development of a web-based genetics institute for a nursing audience. J Contin Educ Nurs. 2004; 35:223-231.

40. Whitt KJ. Experiences from the National Institute of Nursing Research: Summer Genetics Institute 2004. Policy Polit Nurs Pract. 2005;6:15-16

41. Tonkin E, Calzone K, Jenkins J, et al. Genomic education resources for nursing faculty. J Nurs Scholarsh. 2011;43:330-340.

42. Williams JK, Prows CA, Conley YP, et al. Strategies to prepare faculty to integrate genomics into nursing education programs. J Nurs Scholarsh. 2011:43:231-238.

43. Jenkins J, Calzone KA. Genomics Nursing Faculty Champion Initiative. Nurse Educ. 2014;39:8-13.

44. Read CY, Ward LD. Faculty performance on the Genomic Nursing Concept Inventory. J Nurs Scholarsh. 2016;48:5-13.

45. American Nurses Association and International Society of Nurses in Genetics Genetics/genomics nursing: scope and standards of practice. 2nd ed. Silver Spring, MD: Nursebooks.org; 2016.

46. International Society of Nurses in Genetics (ISONG). https://www.isong. org/. Accessed 19 January 2019.

47. Calzone KA, Jenkins J, Prows CA, Masny A. Establishing the outcome indicators for the essential nursing competencies and curricula guidelines for genetics and genomics. J Prof Nurs. 2011; 27:179-191

48. Greco KE, Tinley S, Seibert D. (2011). Development of the essential genetic and genomic competencies for nurses with graduate degrees. Annu Rev Nurs Res. 2011;29:173-190.

49. American Association of Colleges of Nursing. AACN essentials. 2018. https://www.aacnnursing.org/Education-Resources/AACN-Essentials. Accessed 19 January 2019

50. National Council of State Boards of Nursing. Report of findings from the 2014 RN Nursing Knowledge Survey. Chicago, IL: 2015.

51. Jenkins J, Calzone KA, Caskey S, et al. Methods of genomic competency integration in practice. J Nurs Scholarsh. 2015;47:200-210.

52. Martsolf GR, Barnes $H$, Richards MR, et al. Employment of advanced practice clinicians in physician practices. JAMA Intern Med. 2018;178:988-990.

53. Martsolf GR, Nguyen P, Freund D, Poghosyan L. What we know about postgraduate nurse practitioner residency and fellowship programs. J Nurs Pract. 2017;13:482-487.

54. American Academy of Physician Assistants. What is a PA? 2018. https:// www.aapa.org/what-is-a-pa/. Accessed 23 November 2018.

55. Goldgar C, Rackover M. Current status of genetics education and needs assessment of physician assistant programs: a nationwide survey. J Physician Assist Educ. 2007;18:54-60.
56. Rackover M, Goldgar C, Wolpert C, et al. Establishing essential physician assistant clinical competencies guidelines for genetics and genomics. J Physician Assist Educ. 2007;18:47-48.

57. Goldgar C, Michaud NP, Jenkins J. Physician assistant genomic competencies. J Physician Assist Educ. 2016;27:110

58. American Academy of Physician Assistants. Special Interest Groups, Society of PAs in Genetics and Genomics. 2018. https://www.aapa.org/ about/constituent-organizations/special-interest-groups/group/ 810656475/. Accessed 23 November 2018.

59. City of Hope. Cancer Genomics Education Program. 2018. https://www. cityofhope.org/education/health-professional-education/cancergenomics-education-program. Accessed 23 November 2018.

60. Next Generation Science Standards. 2019. https://www. nextgenscience. org. Accessed 19 January 2019

61. The Jackson Laboratory. Teaching the genome generation-New England. https://www.jax.org/education-and-learning/education-calendar/2018/ june/teaching-the-genome-generation. Accessed 8 February 2019.

62. Genomics Education Partnership | Genomics Research. http://gep.wustl. edu. Accessed 19 January 2019.

63. American Society of Human Genetics. Careers in human genetics. https:// www.ashg.org/education/careers.shtml. Accessed 19 January 2019.

64. Genetics/Genomics Competency Center. http://genomicseducation.net. Accessed 19 January 2019

65. Association of Professors of Human and Medical Genetics. http://www. aphmg.org. Accessed 19 January 2019

66. Center for the Integration of Research, Teaching and Learning. http:// www.cirtl.net. Accessed 19 January 2019.

67. The Jackson Laboratory. Education and learning. https://www.jax.org/ education-and-learning. Accessed 19 January 2019.

68. The American Medical Association Education Center. Precision medicine: a tailored approach to health care that accounts for individual variability in genes, environment, and lifestyle. https://education.ama-assn.org/ precision-medicine.html. Accessed 25 March 2019

69. ten Cate O, Scheele F. Competency-based postgraduate training: can we bridge the gap between theory and clinical practice? Acad Med. 2007;82:542-547.

70. Korf BR, Berry $A B$, Limson $M$, et al. Framework for development of physician competencies in genomic medicine: report of the Competencies Working Group of the Inter-Society Coordinating Committee for Physician Education in Genomics. Genet Med. 2014;16:804.

71. National Human Genome Research Institute (NHGRI). Inter-Society Coordinating Committee for Practitioner Education in Genomics (ISCC). 2018. https://www.genome.gov/27554614/intersociety-coordinatingcommittee-for-practitioner-education-in-genomics-iscc/\#al-2. Accessed 8 February 2019. 